\title{
TRAINING FOR DESIGN OF EXPERIMENTS USING A CATAPULT
}

\author{
JIJU ANTONY* \\ International Manufacturing Centre, Warwick Manufacturing Group, University of Warwick, Coventry CV4 7AL, UK
}

\begin{abstract}
SUMMARY
Design of experiments (DOE) is a powerful approach for discovering a set of process (or design) variables which are most important to the process and then determine at what levels these variables must be kept to optimize the response (or quality characteristic) of interest. This paper presents two catapult experiments which can be easily taught to engineers and managers in organizations to train for design of experiments. The results of this experiment have been taken from a real live catapult experiment performed by a group of engineers in a company during the training program on DOE. The first experiment was conducted to separate out the key factors (or variables) from the trivial and the second experiment was carried out using the key factors to understand the nature of interactions among the key factors. The results of the experiment were analysed using simple but powerful graphical tools for rapid and easier understanding of the results to engineers with limited statistical competency. Copyright @ 2002 John Wiley \& Sons, Ltd.
\end{abstract}

KEY WORDS: design of experiments; training; screening; interactions; factorial experiments

\section{INTRODUCTION}

Design of experiments (DOE) is a powerful technique used for discovering a set of process variables (or factors) which are most important to the process (or system) and then determine at what levels these factors must be kept to optimize the process (or system) performance [1]. It provides a quick and cost-effective method to understand and optimize any manufacturing processes. It is a direct replacement of the hit or miss approach of experimentation which requires a lot of guess work and luck for its success in real life situations. Moreover, the hit or miss approach does not take into account interactions among the factors (or variables) and therefore there is always a risk of arriving at false optimum conditions for the process under investigation.

In the past decade or so, DOE has gained increasing importance in the reduction of variability in core processes, whereby consistent product quality can be achieved [2-5]. Moreover, companies striving for a six-sigma approach to achieving quality treats DOE as the key player. The author believes that DOE must be a key element of the management strategy in the 21st century in many manufacturing companies during new product and process introduction, so that robust and

\footnotetext{
*Correspondence to: Jiju Antony, International Manufacturing Centre, Warwick Manufacturing Group, University of Warwick, Coventry CV4 7AL, UK. Email: Jiju.Antony@warwick.ac.uk
}

consistent performance of the product can be achieved in the user's environment.

The purpose of this paper is to provide an insight into the process of understanding the role of DOE on the part of a group of engineers and managers with the help of two catapult experiments. The results of the experiment have been extracted from real catapult experiments performed by a group of engineers in a company during a training program on DOE. The first experiment was performed to identify the key variables or factors which affect the response (in-flight distance) of interest. This is called a screening experiment and the objective is to separate out the key factors (or variables) from the trivial. Having identified the key factors, the team performed a second experiment with the object of understanding the nature of interactions (if present) among the key factors. The results of the experiment were analysed using Minitab software for rapid and easier understanding of the results.

\section{SCREENING EXPERIMENT}

In many new product and process development applications, the number of potential input factors or variables is often excessively large. Under such circumstances, screening experiments are useful to reduce the number of variables to a manageable size so that further experiments can be performed using 
Table 1. List of factors and their levels considered for the screening experiment

\begin{tabular}{lccc}
\hline Control factors & Labels & Low level setting (-1) & High level setting (+) \\
\hline Ball type & A & Pink (P) & Yellow (Y) \\
Rubber band type & B & Brown (BR) & Black (BL) \\
Stop position & C & 1 & 4 \\
Peg height & D & 1 & 4 \\
Hook position & E & 1 & 4 \\
Cup position & F & 5 & 6 \\
Release angle & G & Full & 170 \\
\hline
\end{tabular}

these key variables for a better understanding of the process/product. This reduction strategy in the number of variables allows experiments to focus process or product development/improvement efforts on the key factors or variables.

In manufacturing organizations, generally twolevel fractional factorial designs or Plackett-Burmann (P-B) designs are used with the object of separating the 'vital few' from the 'trivial many' variables or factors. In this paper, the author discusses the $\mathrm{P}-\mathrm{B}$ designs which have been used for teaching the screening experiments during a training programme on DOE within a world-class company. The rationale behind the selection of $\mathrm{P}-\mathrm{B}$ designs for this experiment was to identify the most important factors from the unimportant ones. The object here was not to study all the main and interaction effects; rather it was to determine the key main effects only. These designs were first introduced by Plackett and Burmann [6], and are suitable for studying $k=(N-1) /(L-1)$, where $k$ is the number of factors, $L$ is the number of levels and $N$ is the number of trials/runs. The P-B designs are based on Hadamard matrices in which the number of runs is a multiple of 4 , i.e. $N=4,8,12,16$ and so on. These designs generally do not allow experimenters to study the interactions among the factors/process variables. For screening experiments, we generally maintain the number of levels for the factors at two, in order to keep the experimental budget and resources at a minimum.

For the catapult experiment, the first step was to formulate the problem or to determine the objective of the experiment so that everyone in the team has a common understanding of what needs to be accomplished by the first experiment. There were about six people in the group and all of them were engineers with more than four years experience (on average) with the process. The role of the author was to facilitate the brainstorming session in terms of identification of the factors which affect the response (or output). The response of interests was in-flight

Copyright $\odot 2002$ John Wiley \& Sons, Ltd.
Table 2. Experimental layout for the screening experiment based on $\mathrm{P}-\mathrm{B}_{12}$. ( ) represents the experimental runs in random order.

\begin{tabular}{lllllllll}
\hline Run & A & B & C & D & E & F & G & $\begin{array}{c}\text { Distance } \\
(\mathrm{cm})\end{array}$ \\
\hline $1(12)$ & Y & BR & 4 & 1 & 1 & 5 & 170 & 119 \\
$2(3)$ & Y & BL & 1 & 4 & 1 & 5 & Full & 161 \\
$3(1)$ & P & BL & 4 & 1 & 4 & 5 & Full & 253 \\
$4(5)$ & Y & BR & 4 & 4 & 1 & 6 & Full & 249 \\
$5(11)$ & Y & BR & 1 & 4 & 4 & 5 & 170 & 114 \\
$6(2)$ & Y & BL & 4 & 1 & 4 & 6 & Full & 227 \\
$7(7)$ & P & BL & 4 & 4 & 1 & 6 & 170 & 214 \\
$8(10)$ & P & BR & 4 & 4 & 4 & 5 & 170 & 327 \\
$9(8)$ & P & BR & 1 & 4 & 4 & 6 & Full & 304 \\
$10(4)$ & Y & BR & 1 & 1 & 4 & 6 & 170 & 60 \\
$11(9)$ & P & BL & 1 & 1 & 1 & 6 & 170 & 18 \\
$12(6)$ & P & BR & 1 & 1 & 1 & 5 & Full & 78 \\
\hline
\end{tabular}

distance in centimetres. The following factors and levels were identified from brainstorming which might influence the in-flight distance (refer to Table 1).

Having identified the factors and their levels, the next step was to select a suitable P-B design for studying all the seven factors. In order to create some degrees of freedom for the error term, it was decided to select $\mathrm{P}-\mathrm{B}_{12}$ (i.e. the 12-run $\mathrm{P}-\mathrm{B}$ design), which has eleven degrees of freedom. As each factor was studied at two levels of screening experiments, the degrees of freedom associated with the factors was seven. This clearly satisfies the criterion that the number of experimental trials required for an experiment must be greater than the degrees of freedom associated with the main effects and the interaction effects (if any) to be studied for the experiment [7]. The remaining four degrees of freedom are used to estimate the error variance so that we can make reliable and sound conclusions on the significance of factor effects. The experimental layout for the first experiment which shows all the factor settings is shown in Table 2. The last column in the table represents the in-flight distance corresponding to each trial condition.

Qual. Reliab. Engng. Int. 2002; 18: 29-35 
(response is Distance, Alpha $=.10$ )

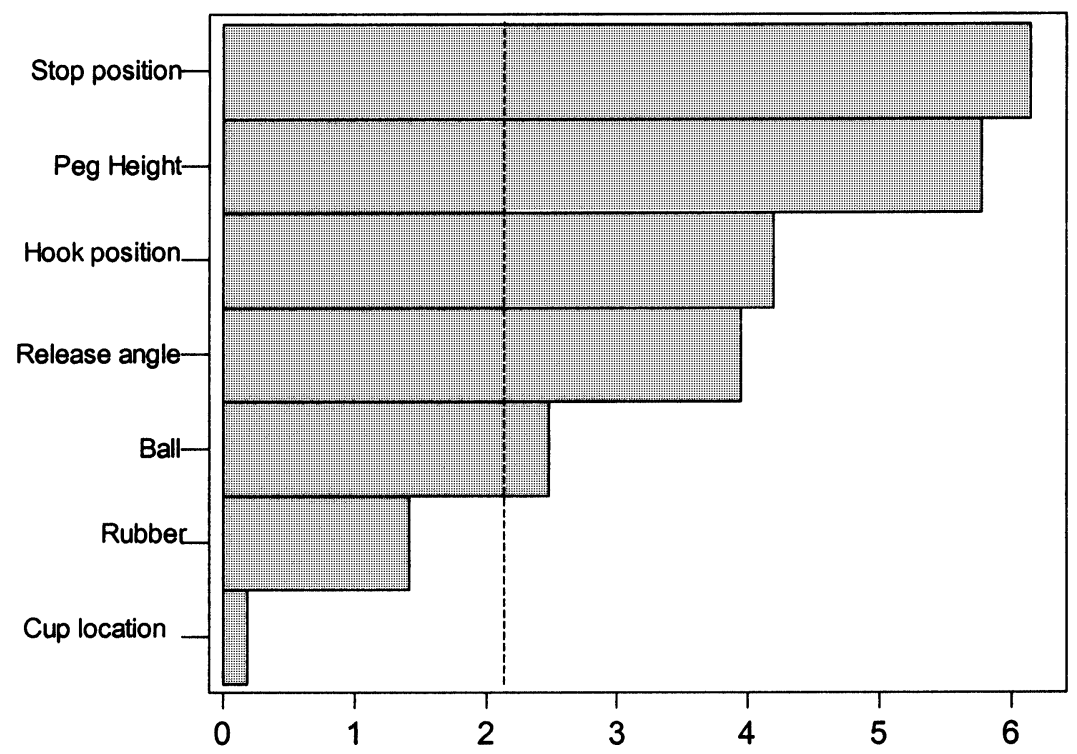

Figure 1. Pareto plot of the main effects from the screening experiment

Having obtained the response (in-flight distance) values, the next was to analyse and interpret the results so that necessary actions can be taken accordingly. The analysis of the experiment is often dependent on its objective. In this case, the objective was to identify the key factors which affect the in-flight distance. The team used Minitab to analyse the data from the experiment. A Pareto plot of the effects was constructed to plot the effects in decreasing order of the absolute value of the standardized effects and draw a reference line on the chart. Any effect that extends past this reference line is considered to have a significant impact on the response. The Pareto plot (Figure 1) shows that factors stop position, peg height, hook position, release angle and type of ball are significant at a significance level of $10 \%$. Factors such as type of rubber and cup position seemed to have no influence on the inflight distance. Moreover, the type of ball is a qualitative factor and the analysis shows that the average distance is higher at low level setting (i.e. pink ball) compared to high level setting (i.e. yellow ball). Therefore it was decided to keep the type of ball (i.e. pink ball) constant for subsequent experiments.

\section{FACTORIAL EXPERIMENT}

A factorial experiment is an experiment where one may vary all the factors in the experiment at their respective levels simultaneously [8]. A factorial experiment can be either full factorial or fractional factorial. A full factorial experiment is generally represented by $2^{k}$, where $k$ is the number of factors. A full factorial experiment in industrial situations is generally suitable for studying up to four factors. If the number of factors is more than four, fractional factorial experiments can be utilized. A fractional factorial experiment is generally represented by $\left(2^{(k-p)}\right)$, where $1 /\left(2^{p}\right)$ represents the fraction. For more information on fractional factorial experiments, the readers are advised to consult [9]. From the screening experiment, the team has identified four important or key factors: stop position, peg height, hook position and release angle. It was decided to perform a full factorial experiment using these four factors. The cup position was kept at the 6th position throughout the experiment as it yielded higher mean in-flight distance from the previous experiment. Similarly brown rubber band was used throughout the experiment, as the mean in-flight distance for brown band was comparatively higher than black rubber band. Table 3 shows the list of factors and their levels for the full factorial experiment. The experiment was replicated twice to capture the variation due to set up and air flow in the room. Each trial or run was randomized to minimize the effect of noise. Randomization is a method of safeguarding the experiment from systematic bias which causes variations in response or output. The results of the full factorial experiment are shown in Table 4. 
Table 3. List of factors and their levels for the full factorial experiment

\begin{tabular}{lccc}
\hline Control factors & Labels & $\begin{array}{c}\text { Low-level } \\
\text { setting }\end{array}$ & $\begin{array}{c}\text { High-level } \\
\text { setting }\end{array}$ \\
\hline Release angle & A & 180 & Full \\
Peg height & B & 3 & 4 \\
Stop position & C & 3 & 5 \\
Hook position & D & 3 & 5 \\
\hline
\end{tabular}

Table 4. Experimental results of full factorial experiment

\begin{tabular}{|c|c|c|c|c|c|c|}
\hline Run & A & B & C & D & \multicolumn{2}{|c|}{$\begin{array}{l}\text { Distance } \\
(\mathrm{cm})\end{array}$} \\
\hline 1 & 180 & 3 & 3 & 3 & 363 & 364 \\
\hline 2 & Full & 3 & 3 & 3 & 401 & 406 \\
\hline 3 & 180 & 4 & 3 & 3 & 416 & 460 \\
\hline 4 & Full & 4 & 3 & 3 & 470 & 490 \\
\hline 5 & 180 & 3 & 5 & 3 & 380 & 383 \\
\hline 6 & Full & 3 & 5 & 3 & 437 & 440 \\
\hline 7 & 180 & 4 & 5 & 3 & 474 & 477 \\
\hline 8 & Full & 4 & 5 & 3 & 532 & 558 \\
\hline 9 & 180 & 3 & 3 & 5 & 426 & 413 \\
\hline 10 & Full & 3 & 3 & 5 & 474 & 494 \\
\hline 11 & 180 & 4 & 3 & 5 & 480 & 502 \\
\hline 12 & Full & 4 & 3 & 5 & 520 & 555 \\
\hline 13 & 180 & 3 & 5 & 5 & 446 & 467 \\
\hline 14 & Full & 3 & 5 & 5 & 512 & 550 \\
\hline 15 & 180 & 4 & 5 & 5 & 480 & 485 \\
\hline 16 & Full & 4 & 5 & 5 & 580 & 591 \\
\hline
\end{tabular}

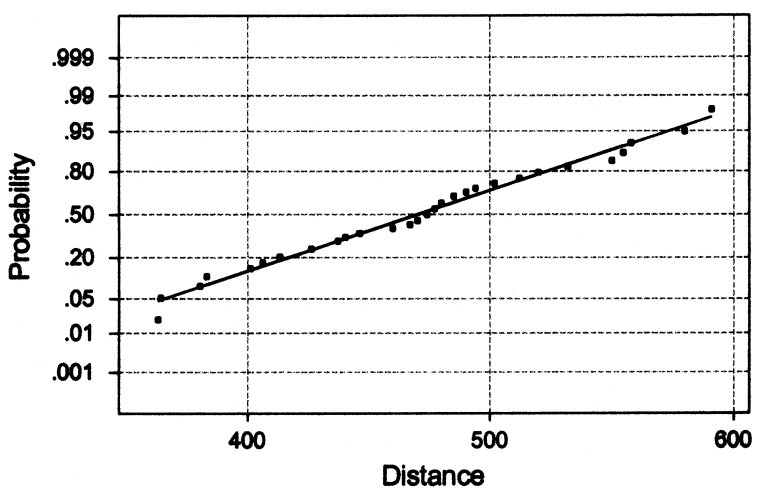

Figure 2. Normal probability plot of data from a full factorial experiment

Prior to carrying out any statistical analysis, the first step was to check the data for normality assumptions. An Anderson-Darling test was performed on the data (Figure 2) which clearly indicates that the data fairly follows a normal distribution. This is also verified and justified by constructing a normal probability plot of residuals (i.e. observed values-predicted values).

Copyright $\odot 2002$ John Wiley \& Sons, Ltd.
All the points on the normal plot come close to form a straight line which implies that the data are fairly normal.

\section{STATISTICAL ANALYSIS AND INTERPRETATION RESULTS}

Having obtained the results of the experiment, the first step was to compute the main and interaction effects of control factors. The main effect of a control factor is the difference in average response observed due to a change in the level of the factor (i.e. change from level 1 to level 2 in this case). The main effects plot for the experiment is shown in Figure 3. The use of main effects plot is to determine which set of factors influence the response and to compare the relative strength of the effects. In a main effects plot, we plot the mean response (i.e. mean distance in this case) at each factor level and then connect the points by a straight line. A reference line is drawn at the overall mean of observations. If the slope of the line connecting the average responses for a factor is parallel to the $x$-axis, then it implies that there is no main effect present. The greater the slope of the line, the stronger the main effect.

Figure 3 indicates that peg height is the most important control factor which affects the mean in-flight distance, followed by release angle, hook position and stop position. In order to determine whether or not these main effects and the interactions among them are statistically significant, it was decided to use a Pareto plot using Minitab software [10]. Minitab plots the effects in decreasing order of the absolute value of the standardized effects and draws a reference line on the chart. Any effect that extends past this reference line is significant. The Pareto plot of the effects shows (Figure 4) that all the main effects (A, B, C and D) and one interaction effect (BD) are statistically significant at $1 \%$ significance level.

Two factors are said to interact with each other if the effect of one factor on the response is different at different levels of the other factor [11]. In order to interpret interaction between B (peg height) and D (hook position), it is best to construct an interaction plot. If the lines in the plot are parallel to each other, no interaction exists between the factors. This implies that the change in the mean response from low to high level of a factor does not depend on the level of the other factor. If the lines are non-parallel, an interaction exists between the factors. The greater the degree of departure from being parallel, the stronger the interaction effect. Figure 5 illustrates the interaction plot between B and D.

Qual. Reliab. Engng. Int. 2002; 18: 29-35 


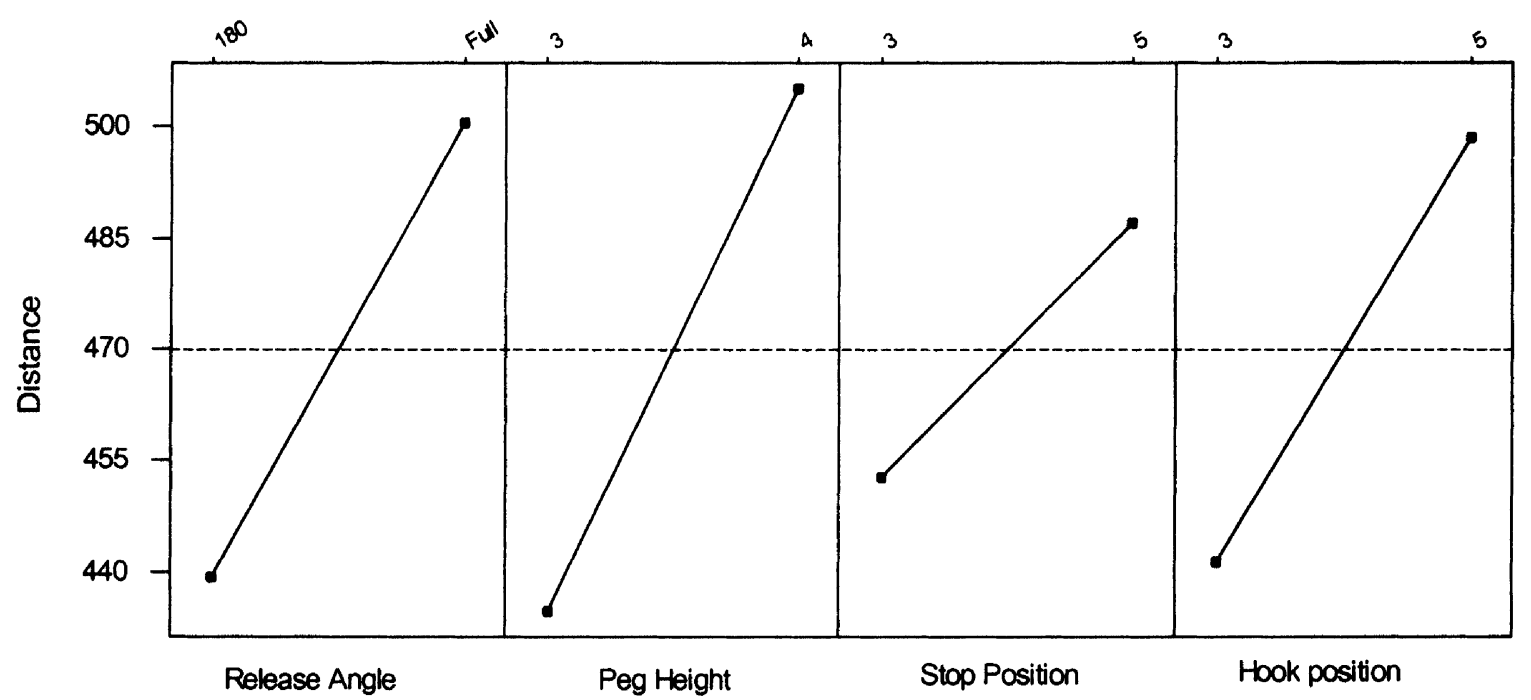

Figure 3. Main-effects plot for the full factorial experiment

(response is Distance, Alpha $=.01$ )

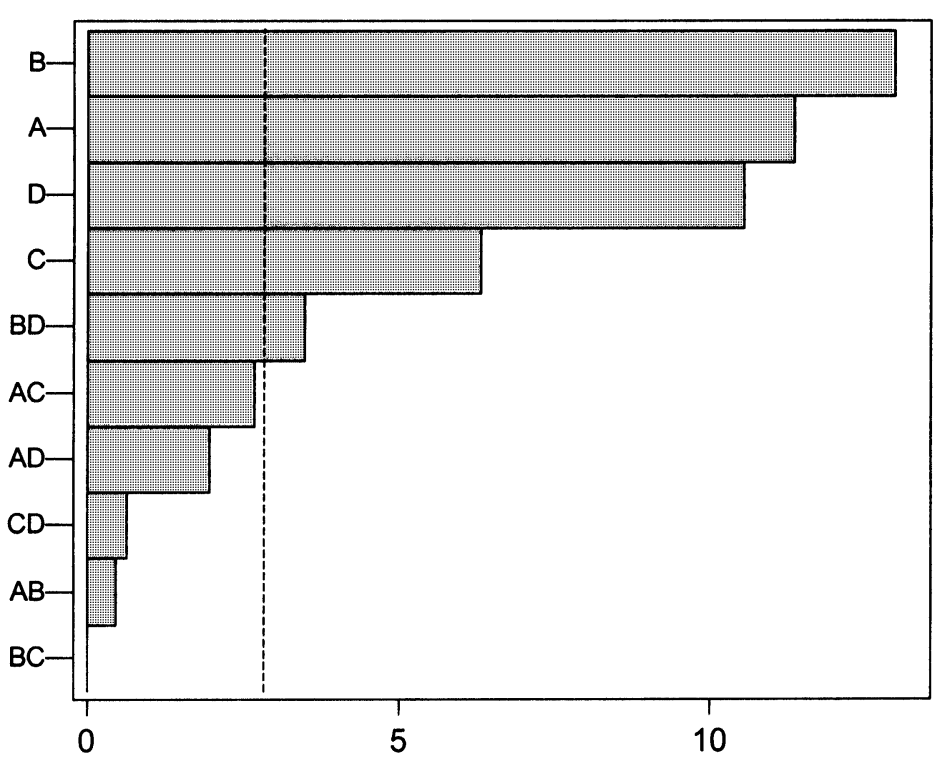

A: Release angle

B: Peg Height

C: Stop Position

D: Hook position

Figure 4. Pareto plot of the effects (main and interaction effects)

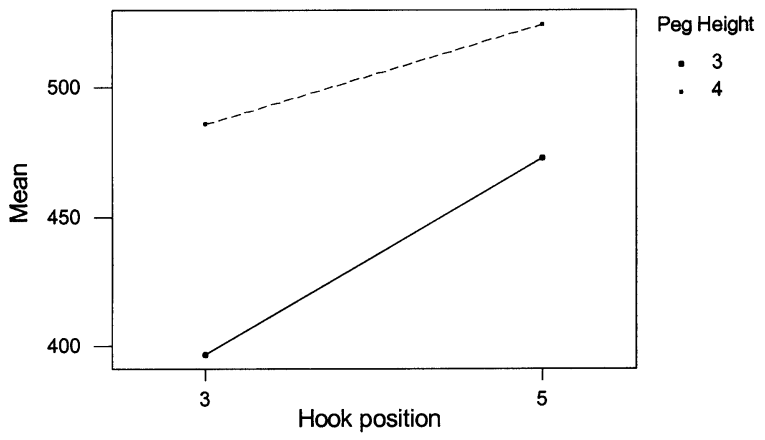

Figure 5. Interaction plot between hook position and peg height

Copyright $@ 2002$ John Wiley \& Sons, Ltd.
The above plot shows that the effect of hook position on the distance is different at different levels of peg height position. This clearly implies that there is an interaction between these two factors. The graph shows that maximum in-flight distance is achieved when hook position is kept at position 5 and peg height at position 4 .

\section{DETERMINATION OF OPTIMAL CONTROL FACTOR SETTINGS}

Having obtained the significant control factors and the interactions among them, the next step is to determine 
Table 5. Final optimal control factor settings

\begin{tabular}{lc}
\hline Control factors & Optimal level \\
\hline Release angle & Full \\
Peg height & 4 \\
Stop position & 5 \\
Hook position & 5 \\
\hline
\end{tabular}

the optimal settings that will maximize the in-flight distance. In order to arrive at the optimal condition, the mean in-flight distance at each level of the control factors was analysed. As interaction between $\mathrm{C}$ and $\mathrm{D}$ appeared to be significant, it was also important to determine the combination of $\mathrm{C}$ and $\mathrm{D}$ which yield maximum in-flight distance. Table 5 illustrates the optimal settings of control factors. The optimal combination of factors may be obtained from Figure 3 .

It is quite interesting to note that the optimal condition is one which corresponds to trial condition 16 (Table 4). A confirmatory experiment was carried out to verify the results from the analysis. Five readings were made based on the optimal combination of control factors. The average in-flight distance was estimated to be $584 \mathrm{~cm}$. It was also found that a change of stop position from 5 to 4 has yielded even better average results $(596 \mathrm{~cm})$.

\section{SIGNIFICANCE OF THE WORK}

The purpose of this section is to bring the significance of teaching experimental design techniques to engineers using simple but powerful graphical tools. The focus was to minimize the mathematical aspects of experimental design and to bring modern graphical tools for rapid decision-making processes. The engineers in the company felt that the experiments were useful in terms of formulating the problem, identifying the key control factors, determining the ranges of factor settings, selecting the experimental layout, assigning the control factors to the design matrix, conducting the experiment, and analysing and interpreting the results of the experiment. The author believes that experiments of such nature will assist engineers to design experiments in their own work environment.

\section{CONCLUSIONS}

DOE is a powerful approach to problem solving in many production processes. It is a technique for improving the process stability and capability, yield and performance of on-going manufacturing

Copyright $\odot 2002$ John Wiley \& Sons, Ltd. processes. The purpose of this paper has been to illustrate the importance of screening experiments and full factorial experiments using two catapult experiments. Screening experiments using P-B design were used to identify the most important control factors. P-B designs were used to separate out the 'vital few' main effects from the 'trivial many'. Having identified the key factors from the screening experiment, a full factorial experiment was used to study both the main effects and the interaction effects.

Apart from the use of $\mathrm{P}-\mathrm{B}$ designs for screening experiments, one can also look at the use of powerful saturated fractional factorial designs. Instead of using a 12-run $\mathrm{P}-\mathrm{B}$ design, it is also worthwhile looking at the use of $2^{(7-4)}$ fractional factorial design for studying seven factors in eight trials. This is a resolution III design, where main effects are confounded with two-way interaction effects. Design resolution is a summary characteristic of confounding patterns [12]. In order to study the main effects clear of two-way interactions, one could utilize the use of fold-over designs or reflected designs advocated by Box [9].

The analysis was carried out using simple but powerful modern graphical tools such as a main effects plot, an interaction plot and a normal probability plot. In order to keep the experiment simple, two levels were used for the study. The results of these experiments have provided a stimulus for the wider application of experimental design techniques by engineers within the organization in real-life situations.

\section{REFERENCES}

1. Antony J, Kaye M. Experimental Quality: A Strategic Approach to Achieve and Improve Quality. Kluwer Academic: New York, 1999.

2. Sirvanci MB, Durmaz K. Variation reduction by the use of designed experiments. Quality Engineering 1993; 5(4):611618.

3. Schmidt S, Launsby RG. Understanding Industrial Designed Experiments. Air Academy Press: CO, 1992.

4. Antony $\mathrm{J}$ et al. Reducing manufacturing process variability using experimental design technique: A case study. Integraded Manufacturing Systems 1999; 10(3/4):162-169.

5. Peace GS. Taguchi Methods-A Hands on Approach. AddisonWesley: MA, 1993.

6. Plackett RL, Burmann JP. The design of optimum multifactorial experiments. Biometrika 1946; 33:305-325.

7. Ross PJ. Taguchi Techniques for Quality Engineering. McGraw-Hill, 1988

8. William GW. Experimental design: Robustness and power issues. ASQ Congress Transactions 1990; 1051-1056.

9. Box GEP et al. Statistics for Experimenters. Wiley: New York, 1978.

10. Minitab User's Guide, Data Analysis and Quality Tools, Release 13. Minitab, Inc., 2000, UK.

Qual. Reliab. Engng. Int. 2002; 18: 29-35 
11. Antony J, Antony FJ. Teaching advanced statistical techniques to industrial engineers and business managers. Journal of Engineering Design 1998; 9(1):89-100.

12. Antony J. A strategic methodology for the use of advanced statistical quality improvement techniques. PhD Thesis, University of Portsmouth, UK, 1997.

\section{Author's biography:}

Jiju Antony is a Senior Teaching Fellow at the International Manufacturing Centre of the University of Warwick. $\mathrm{He}$ received a $\mathrm{BE}$ in Mechanical Engineering from the University of Kerala, South India, MEngSc in Quality and Reliability Engineering from the National University of Ireland and a PhD in Quality Control for Manufacturing from the University of Portsmouth. $\mathrm{He}$ is a member of the Institute of Quality Assurance and American Society for Quality. He has published over 50 refereed papers in the areas of Reliability Engineering, Design of Experiments, Taguchi Methods, Total Quality Management, Statistical Process Control and Six Sigma. His primary research areas include Design of Experiments and Taguchi Methods for Improving Process Quality, Robust Technology Development, Statistical Process Control for Variability Reduction, Problem Solving using Quality Tools and Techniques and Six Sigma. He has co-authored a book entitled 'Experimental Quality: A Strategic Approach to Achieve and Improve Quality' published by Kluwer Academic Publishers in December 1999. His second edited book entitled 'Understanding, Managing and Implementing Quality: Tools, Techniques and Frameworks' is due to be published by Routledge publishers in December 2001. 